\title{
A DIVIDE-AND-CONQUER APPROACH FOR DENOISING AND MODELING THE CN TOWER LIGHTNING CURRENT DERIVATIVE SIGNAL
}

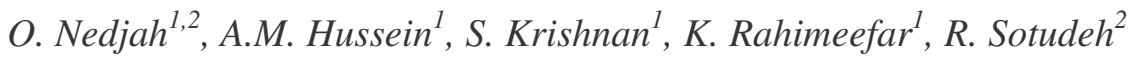 \\ ${ }^{1}$ Electrical and Computer Engineering Department, Ryerson University, Toronto, Ontario, Canada \\ ${ }^{2}$ Communication and Electrical Engineering, Hertfordshire University, Hatfield, Hertfordshire, U.K.
}

\begin{abstract}
The CN Tower is a transmission hub and an instrumented tower for the measurement of the lightning return stroke current derivative. The recorded data are corrupted by different kinds of noise, and need to be denoised for accurate determination of the lightning return-stroke current waveform parameters. A new Divide-and-Conquer denoising approach that imitates the Basis Pursuit method and the Newton-Raphson technique has been developed. This paper describes the new process of denoising the recorded signals. First, the current derivative is preprocessed to eliminate the noise outside the lightning return-stroke active region and reduce the presence of the high frequencies inside the active region. Then, by marching on both the graphs of the current derivative and its integral, the noise due to reflections is localized and removed. By this process the SNR improved by $35 \mathrm{~dB}$ and the lightning current and current derivative parameters are calculated automatically with a high precision. Furthermore, using the calculated parameters the data is curve fitted to Heidler function, which results in a model for the measured lightning current derivative with an infinite SNR.
\end{abstract}

Index Terms - Lightning current derivative, denoising methods, Heidler function.

\section{INTRODUCTION}

Lightning is defined as a transient high current electric discharge. It occurs when some region of the atmosphere gains such a large charge that the electric fields associated with it can cause an electric breakdown of the air [1]. These transient high currents reaching the earth can be devastating to modern societies. They frequently cause blackouts and they can destroy or interrupt the operations of communication networks, aircrafts, spacecrafts, electric and electronic systems. Protection from its hazards made of the lightning discharge an important area of research in many institutions from the seventies. Researchers are also interested in getting a better understanding of the lightning phenomenon itself. Also the studies are meant to get a deep knowledge of the discharged currents and the induced and radiated electromagnetic fields in the vicinity of the discharge especially on tall structures. This knowledge will help to design adequate protection systems for the communication and transmission lines systems.

The protection systems are designed based on statistical data recorded by lightning measurement systems. These data has to be accurate for the designed protection systems to be efficient.

Lightning strikes to the Canadian National (CN) Tower have been observed since 1978. Since 1991, several measurement stations have been in operation to simultaneously capture the lightning current derivative at the $\mathrm{CN}$ Tower and its associated lightning-generated electromagnetic pulse $2 \mathrm{~km}$ north of the tower, the lightning trajectory images taken from two orthogonal directions, and the return-stroke velocity [2-4].

CCECE/CCGEI May 5-7 2008 Niagara Falls. Canada $978-1-4244-1643-1 / 08 / \$ 25.00$ (C) 2008 IEEE
The $\mathrm{CN}$ tower lightning measurement systems provide valuable statistical data to the lightning research and protection communities.

The systems protection community is especially interested in the knowledge of the lighting current and current derivative waveforms parameters that are; the current wavefront peak, the maximum current wavefront steepness, the $10-90 \%$ risetime to the current peak, and the pulse width at the $50 \%$ level of the peak

As the $\mathrm{CN}$ Tower main function is a transmission hub and as it it is built by the adjunction of many parts, the measurements of the lightning current derivative at the tower are noisy. Because of the existence of different kinds of noise, the extraction of the lightning current waveform parameters is difficult and the results are in many cases inaccurate.

The noise includes high frequencies due to the function of the tower as a transmission utility, a DC offset due to the measurement system, Loran-C frequencies [5], and the interference of reflected currents due to the tower's structural discontinuities.

A brief description of the lightning current derivative measurement system housed at the tower is included. Typical current derivative signals captured at the tower is presented and the difficulties related to the extraction of the current waveform parameters due to the noise will be underlined. The adaptation of the techniques used in the optimization domain for denoising the lightning current derivative and its integral will be discussed, and the results will be emphasized. The noise removal made possible the automatic calculation of the current derivative and the current waveforms parameters, and the generation of a typical Heidler model for every measured signal.

\section{CURRENT MEASUREMENT SYSTEM}

A lightning current derivative measurement system was installed at the CN Tower in 1990. It consists of a 3-m (two $1.5 \mathrm{~m}$-long sections) Rogowski sensing coil having a 40-MHz bandwidth with a sensitivity of $0.35 \mathrm{~V} /(\mathrm{A} / \mathrm{ns})$.. The coil is placed at the $474-\mathrm{m}$ above ground level (AGL) and is connected via a 146-m triaxial cable to a recording system located at the 372-m AGL.

The current derivative recording system consists of a computer controlled $10 \mathrm{~ns}$, 10-bit, two-channel digitizer (Tektronix 710 A). It has a capacity of 128 kilobytes of memory per channel, which enables the recording of up to 8 return strokes in a lightning flash, each lasting $164 \mu \mathrm{s}$.

\section{CURRENT DERIVATIVE SIGNAL}

A lightning flash may contain many strokes. Every stroke is a transient high current electric discharge pulse.

The Rogowski coil measures a voltage signal that is proportional to the lightning current derivative. 
This signal, resulting from a lightning return stroke, is registered in 16 kilobytes of memory by the recording system at a sampling frequency of $100 \mathrm{MHz}$. It forms what is called the returnstroke current derivative waveform. Fig.1 presents a typical lightning return-stroke current derivative signal, measured at the $\mathrm{CN}$ Tower on July 03, 1998 at 17:31 pm. The corresponding lightning current is obtained by numerical integration (Fig. 2).

As seen in Figs. 1-2, the current derivative and its integral signals, are corrupted by different kinds of noise, partly due to the initial function of the $\mathrm{CN}$ Tower as a transmission facility (highfrequency noise). Some of the noise is thought to be due to the measurement system itself, like the DC component. Current reflections due to the tower's structural discontinuities add other interfering noise. Furthermore, a low frequency component oscillating in the vicinity of $100 \mathrm{kHz}$ is always visible in the recorded signals. This low frequency noise component has been proved to be a result of Loran-C signals [6]. As a result of the noise, different frequencies are found in the current derivative waveform, the spectrum of the current derivative waveform of Fig. 1 is shown in Fig. 3.

Depending on the current peak and the current wavefront steepness, the return-stroke lightning current pulse may be distinguishable, slightly exceeding the noise level, or completely embedded in noise [7,8]. Due to the presence of different noise components, Figs. 1-2 demonstrate the difficulty of extracting the current waveform parameters, namely, the wavefront peak, the maximum wavefront steepness, the $10-90 \%$ risetime to the peak and the pulse width at the $50 \%$ level of the peak. In some cases, when the current wavefront steepness and/or the current peak are low, most current waveform parameters, if not all, are impossible to determine.

Several methods have been applied to de-noise the lightning current derivative signals captured at the $\mathrm{CN}$ Tower from the associated noise. We can mention the Linear Filtering, the Fourier Transform based spectral subtraction, the adaptive Wavelet Transforms [7-9] and the optimization based strategies methods that will be described later.

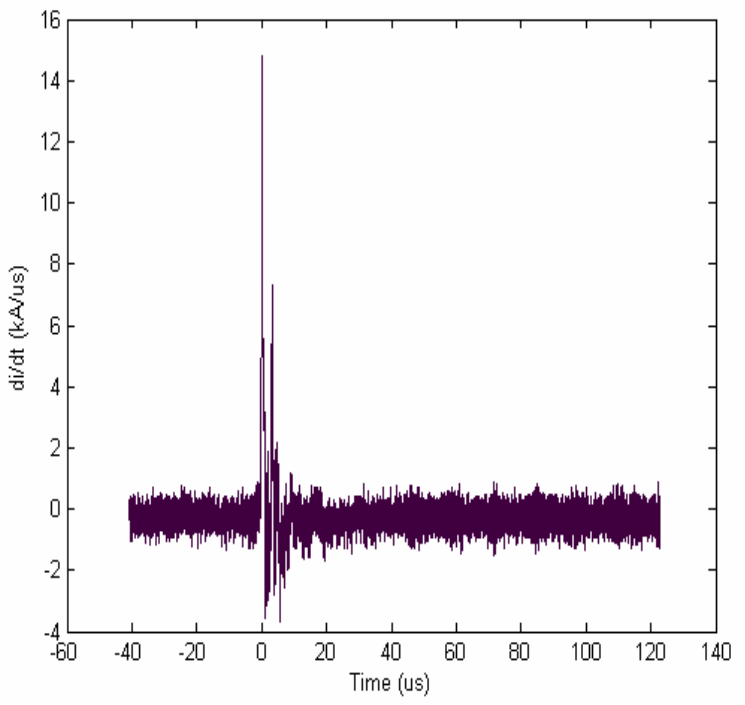

Figure 1. Typical measured current derivative waveform.

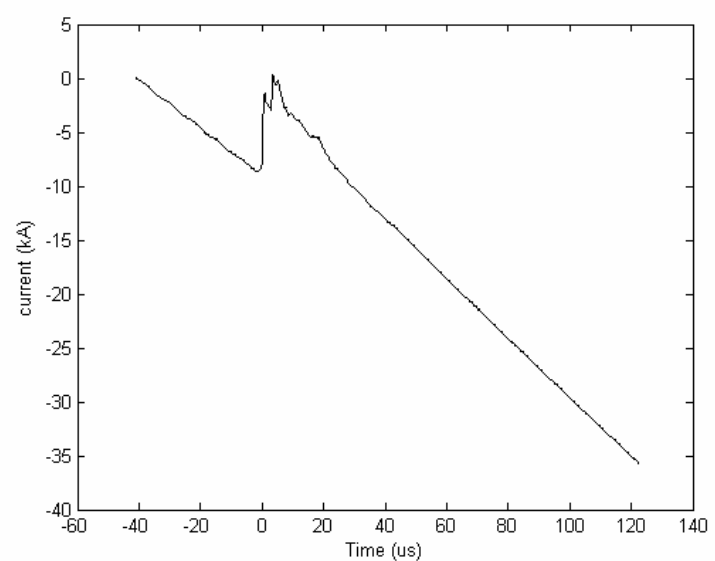

Figure 2. Current waveform.

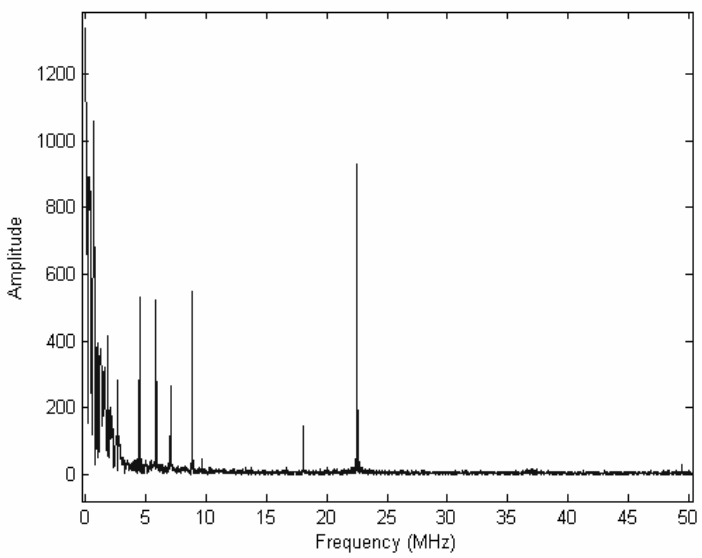

Figure 3. Current derivative spectrum.

\section{HEIDLER MODEL AND ADAPTIVE DENOISE}

\subsection{Heidler Lightning Current Modelisation}

Heidler has developed an empirical form for representing a typical lightning current waveform. This formula has been used in many lightning current related models, including the modified transmission (MTL) model treating lightning strikes to tall structures [10].

The Heidler function is given by the following expression:

$$
i(t)=\frac{I_{1}}{2 c_{1}}\left[\frac{\left(t / \tau_{1,1}\right)^{k_{1}} e^{-t / \tau_{2,1}}}{1+\left(t / \tau_{1,1}\right)^{k_{1}}}\right]+\frac{I_{2}}{2 c_{2}}\left[\frac{\left(t / \tau_{1,2}\right)^{k_{2}} e^{-t / \tau_{2,2}}}{1+\left(t / \tau_{1,2}\right)^{k_{2}}}\right]
$$

Where $I_{i}$ is the current peak, $\left(t / \tau_{1, i}\right)^{k} /\left(1+\left(t / \tau_{1, i}\right)^{k}\right.$ represents the current rise function and $e^{\left(-t / \tau_{2}, i\right)}$ corresponds to the current decay function. $\tau_{1, \mathrm{i}}$ and $\tau_{2, \mathrm{i}}$ are the time constants determining the current rise and decay times, respectively, $\mathrm{k}_{\mathrm{i}}>1$ is a current steepness factor and $c_{i}<1$ is a correction factor [11]. 
After preliminary processing for denoising the measured current derivative waveform the calculated parameters of the current waveform are determined and injected together with Heidler function in a curve fitting process to generate an appropriate model. The generated model represents the measured lightning current derivative with an infinite $S N R$.

\subsection{Denoising Techniques}

A desired measured signal $\mathrm{s}$ is always accompanied by noise during its recording. The general recorded signal can be represented by:

$\mathrm{Y}=\mathrm{s}+\sigma \mathrm{z}$

Where, $\mathrm{z}$ is supposedly a Gaussian $N(0,1)$ additive noise and $\sigma$ the noise level.

The discrete signal $s=\left(s_{t}\right)_{t=0}^{n-1}$ can be approximated by the elements of a dictionary $\mathrm{D},\left(\phi_{\gamma}\right)_{\gamma \in \Gamma}$, where $\gamma$ is a parameter, by the expression:

$s=\sum \alpha_{\gamma_{i}} \phi_{\gamma_{i}}+R^{m}$, where $R^{\mathrm{m}}$ is a residual.

When denoising adaptively a signal we usually represent it in a specific basis according to the adapted method (dictionary elements $\left.\left(\phi_{\gamma}\right)_{\gamma \in \Gamma}\right)$ that can be the Fourier transform, the wavelet transform, the wavelet packet transform, the cosine/sine packet transform, Gabor functions or any other function, and then consider only the components that contribute the most to the energy of the signal, or reject all parts of the signal that are below a specific threshold defined arbitrarily, empirically, or statistically.

Many methods have been developed that stand on this general strategy and differ by the way they are applied, among which we can cite the Best Orthogonal Basis denoising method, the Matching Pursuit strategy, the minimum description length scheme and the Basis Pursuit algorithm that inspired the new developed approach[12-15].

In the Basis Pursuit strategy [15], the signal is decomposed in the wavelet packet, cosine/sine or any other basis, or even in merged dictionary elements. The result of decomposition is put in a matrix format and the matrix representation is optimized to count only the elements that are fundamental to represent the signal, which means the optimum basis.

Hence basis pursuit comes to a matrix reduction. In this method, the problem is formulated and tackled as if it was a linear programming issue by minimizing the $1_{1}$ norm of the representation coefficients to obtain the optimum basis in which the signal can be represented, by solving:

$\left\{\right.$ Minllall $_{1}$

$\{$ subject to $\Phi \alpha=\mathrm{s}$

Translating the denoising problem into the basis pursuit philosophy, the following will be solved:

$\min _{\alpha} \frac{1}{2}\|y-\Phi \alpha\|^{2}+\lambda\|\alpha\|_{1}$

The solution $\alpha(\lambda)$ is a function of the parameter $\lambda$, assuming the dictionary to be normalized so that $\left\|\Phi_{\gamma}\right\|_{2}=1$ for all $\gamma, \lambda$ is set to the value $\lambda_{p}=\sigma \sqrt{2 \log (p)}$, where $\mathrm{p}$ is the cardinality of the dictionary and $\sigma$ is the noise variance. The result of optimization that is the optimum (best) basis is searched via an optimization technique as the simplex method or the primal-dual interior barrier method to look for the basis that best contribute to the signal and reduces the noise[16,17].

As outlined throughout the cited methods a primordial knowledge or guess of the signal characteristics or statistical behavior is necessary for denoising. By tracking the results obtained by the different denoising methods on the lightning current derivative signals, a new strategy proper to the characteristics of the lightning current derivative and its associated current waveform was developed.

The new strategy is built upon the ideas developed in the optimization domain as to divine an initial value for the optimal solution to the problem and to build on it to get to the real optimum one and it rests on the Divide-and-Conquer principle and the Heidler function optimization.

\subsection{Lightning Current Derivative De-noise by Divide-and- Conquer}

As already stated, the measured lightening current derivative signals are contaminated by a wide range of frequencies including a DC component. The removal of the DC part is the first step in the pretreatment of the waveform. Then the range of high frequencies is reduced by an adaptive wavelet transform. This process reduces the number of zero crossings in the current derivative waveform. Since the current waveform is obtained by integration, the high frequency noise is automatically removed from this waveform, which makes the search for the local minima and local maxima on the waveform much easier.

After these preliminary processes, the Divide-and-Conquer process starts based on the initial and final conditions of the lightning signal and the Newton-Raphson optimization technique [17].

The lightning current derivative is divided into three sections; a) the time before the lightning signal, b) the time during the lightning signal, and c) the time after the lightning signal.

As initial and final conditions, it is known that there is no lightning signal before the first appearance of the return stroke; as 20 (40 or 80) $\mu$ s of the total $160 \mu$ s of the signal duration are registered before the triggering process takes place, hence the portion of signal just before this time is equalized to zero.

After the return stroke, other than the peaks reflected from the discontinuities of the tower there should be no more peaks in the lightning current or its derivative, and the current should keep on decaying until reaching the zero value after some milliseconds, so after approximately 10 to $20 \mu \mathrm{s}$ the current derivative signal can be replaced by its mean value, hence reducing more noise.

The maxima and the minima on the current derivative waveform correspond to the maximum steepness of the current waveform respectively on ascending and descending curvatures, and the maxima and the minima on this latter one correspond to the zero-crossing on the current derivative waveform as stated by Newton-Raphson technique. Hence the zero-crossings on the current derivative waveform together with the successive minima and maxima on the current waveform on the active period of the waveforms are tracked simultaneously by marching on the two graphs or set of data.

The maximum current derivative peak is localized, and the first zero crossing of the waveform after it is searched, its position 
corresponds to the first current peak position which corresponds to the lightning channel discharge through the tower.

The maximum peak of the current waveform corresponds to the reflection from the ground. Other small peaks in between the wavefront peak and the ground reflection peak are due to the reflections from the Observation Deck levels of the tower. Without the reflections from the tower discontinuities the current would have been decaying until reaching zero, this is why the peaks following the first peak that are due to the reflections at the discontinuities have to be removed. There are two major reflection points, the reflection from the top of the observation desk and the reflection from the ground. The reflection parameter can be written as the ratio $R=E_{r} / E_{i}$, where $E_{i}$ represents the incident waveform amplitude and $E_{r}$ is the reflected waveform amplitude. Once the first and the second successive highest peaks are localized, the reflection parameters are estimated and the portion of signals related to the reflected waveforms are removed by subtraction. After the current waveform is cleaned, its parameters are calculated automatically.

Together with the Heidler formula, the first three parameters ( the current wavefront peak, the $10 \%$ to $90 \%$ rise time to the peak, its $90 \%$ to $10 \%$ decay time) are injected as initial guesses for the Heidler function parameters in a curve fitting program that is based on the least squares (LSQR) method. The result of optimization represent the Heidler model associated to the lightning current striking the $\mathrm{CN}$ Tower, and the associated current derivative waveform is obtained by differentiation of the current waveform model.

Hence for every measured lightning current derivative waveform we can obtain a clean lightning current waveform with an infinite SNR and that can be represented only by its 10 optimized Heidler function parameters.

The results of application of the developed method on the signal of Fig. 1 are discussed in the next section.

\section{RESULTS}

The signal represented by Figure. 1 has been divided in three sections. The noise corresponding to each section has been removed, By bringing the first section values to zero, filtering out the high frequencies of the active section by the adaptive wavelet transform and replacing the values of the third section by their mean value. Fig. 4 represents the signal of Fig. 1 after these different processes. After denoising the current derivative signal it was possible to localize the different peaks and valleys on the current waveform. These variation points were localized by marching on both the graphs of the current derivative and its integral and tracking simultaneously the zero-crossings and the minima and maxima of both waveforms.

The most important peaks of the current derivative waveform and its integral are shown in the Figure.5. On the figure Ms denotes the peak corresponding to the maximum steepness of the current waveform, $\mathrm{Cp}$ denotes the channel wavefront peak, SkP represents the peak due to the reflected waveform from the top of the Observation Deck, GP refers to the peak of the reflected waveform from the ground and MinC the minimum current between the two peaks corresponding to the reflection from the bottom of the Observation Deck. After the localization of the different reflection peaks the reflection coefficients were estimated and the associated reflected waveforms were removed by subtraction, then the waveform between the reflection peaks was adjusted by linear interpolation. The reflection free current waveform is plotted on the same Fig.5.
Once the noise associated to the measured current derivative and its integral waveforms was reduced it was possible to determine their parameters automatically. The Tables I and II present the manually measured parameters compared to the automatically calculated ones for the current derivative and the current of the signal of Fig.1 and Fig.2. The manual measurements were taken visually on plots of noisy recorded signals where only the DC part of the noise was removed.

The accuracy of the automatic calculation is obvious from the tables. It can also be noticeable in Table II the correction made for the $50 \%$ width and the associated current charge of the current waveform, as the manual calculation includes the width added by the reflections from the Tower, that also affect the total charge evaluation.

For the same signal presented in Fig.1 the $S N R$ of the current derivative waveform was improved from $29.72 \mathrm{~dB}$ to $70.41 \mathrm{~dB}$ after denoising as indicated in Table III.

TABle I. Current derivative Parameters

\begin{tabular}{|l|l|c|c|c|}
\hline $\begin{array}{c}\text { Measurement } \\
\text { method }\end{array}$ & $\begin{array}{c}\text { Max } \\
\text { Peak } \\
(\mathbf{k A} / \boldsymbol{\mu} \mathbf{s})\end{array}$ & $\begin{array}{c}\text { Max } \\
\text { steepness } \\
\left(\mathbf{k A} / \mathbf{\mu s}^{2}\right)\end{array}$ & $\begin{array}{c}\text { Rise } \\
\text { time } \\
(\boldsymbol{\mu} \mathbf{s})\end{array}$ & $\begin{array}{c}\mathbf{5 0 \%} \\
\text { width } \\
(\boldsymbol{\mu} \mathbf{s})\end{array}$ \\
\hline Manual & 14.8 & indefinit & 0.1 & 0.3 \\
\hline Automatic & 14.9 & 321.06 & 0.1 & 0.3 \\
\hline
\end{tabular}

TABLE II. CURRent Parameters

\begin{tabular}{|l|l|l|l|l|c|}
\hline $\begin{array}{c}\text { Measurement } \\
\text { method }\end{array}$ & $\begin{array}{c}\text { Max } \\
\text { Peak } \\
(\mathbf{k A})\end{array}$ & $\begin{array}{c}\text { Max } \\
\text { steepness } \\
(\mathbf{k A} / \boldsymbol{\mu s})\end{array}$ & $\begin{array}{c}\text { Rise } \\
\text { time } \\
(\boldsymbol{\mu s})\end{array}$ & $\begin{array}{c}\mathbf{5 0 \%} \\
\mathbf{w i d t h} \\
(\boldsymbol{\mu s})\end{array}$ & $\begin{array}{c}\text { total } \\
\text { charge } \\
(\mathbf{C})\end{array}$ \\
\hline Manual & 7.7 & 14.8 & 0.7 & 53.6 & 208.6 \\
\hline Automatic & 7.4 & 14.9 & 0.8 & 25.3 & 93.9 \\
\hline
\end{tabular}

The denoised current waveform, the minimal, and maximal permissible values of the lightning current model parameters provided by [18] in Table 1, the calculated lightning current parameters (the wavefront peak value, its rising and falling times) provided as initial guesses for the parameters, together with Heidler function formula were injected into an optimization program. The program consists of a curve fitting process based on the LSQR method provided by Maltlab optimization toolbox. It estimates the heidler model of the measured waveform based on its initial calculated parameters.

The estimated current Heidler model of the signal of Fig.1, that can be considered as the perfect lightning current free of noise is presented in Fig.6. Its associated current derivative model obtained either by evaluating the derivative of Heidler model using the estimated heidler model parameters or simply by differentiating the optimized Heidler current model is shown in Fig.7.

Table.III gives a comparative study between an adaptive wavelet denoising technique that gave the best results relative to the previously described adaptive methods and the Divide-and-Conquer method for signals of different peak ranges.

From Table.III, the supremacy of the Divide-and-Conquer method over the adaptive wavelet technique can be seen. The automatic measured peaks are closer to the manual measured ones in the former one, and the SNR is up to 150 times higher for the 
TABLE III. PEAK AND SIGNAL TO NOISE RATIO COMPARISON BETWEEN ADAPTIVE WAVELET (AW) DENOISING TECHNIQUE VERSUS THE DIVIDE-AND-CONQUER (DAC) DENOISING TECHNIQUE

\begin{tabular}{|l|l|l|l|l|l|l|}
\hline Signal name & $\begin{array}{c}\text { Manual } \\
\text { measured } \\
\text { peak } \\
(\mathbf{k A} / \boldsymbol{\mu} \mathbf{s})\end{array}$ & $\begin{array}{c}\text { Peak of AW } \\
\text { denoised } \\
\text { signal } \\
(\mathbf{k A} / \boldsymbol{\mu} \mathbf{s})\end{array}$ & $\begin{array}{c}\text { Peak of DAC } \\
\text { denoised } \\
\text { signal } \\
(\mathbf{k A} / \boldsymbol{\mu} \mathbf{s})\end{array}$ & $\begin{array}{c}\text { SNR of } \\
\text { original } \\
\text { signal } \\
(\boldsymbol{d} \boldsymbol{B})\end{array}$ & $\begin{array}{c}\text { SNR of AW } \\
\text { denoised } \\
\text { signal } \\
(\boldsymbol{d} \boldsymbol{B})\end{array}$ & $\begin{array}{c}\text { SNR DAC } \\
\text { denoised } \\
\text { signal } \\
(\boldsymbol{d} \boldsymbol{B})\end{array}$ \\
\hline $\mathrm{A} 0270657.701$ & 4.42 & 1.07 & 4.33 & 2. & 12.89 & 167.62 \\
\hline $\mathrm{D} 1145229.902$ & 9.51 & 6.67 & 9.69 & 9.28 & 12.33 & 81.61 \\
\hline $\mathrm{D} 1145230.544$ & 14.8 & 13.9 & 14.9 & 29.72 & 61.41 & 70.41 \\
\hline $\mathrm{D} 1146399.213$ & 24.48 & 23.0 & 24.71 & 27.21 & 35.72 & 79.24 \\
\hline $\mathrm{A} 0280824.252$ & 34.82 & 30.84 & 34.74 & 29.19 & 63.29 & 88.48 \\
\hline
\end{tabular}
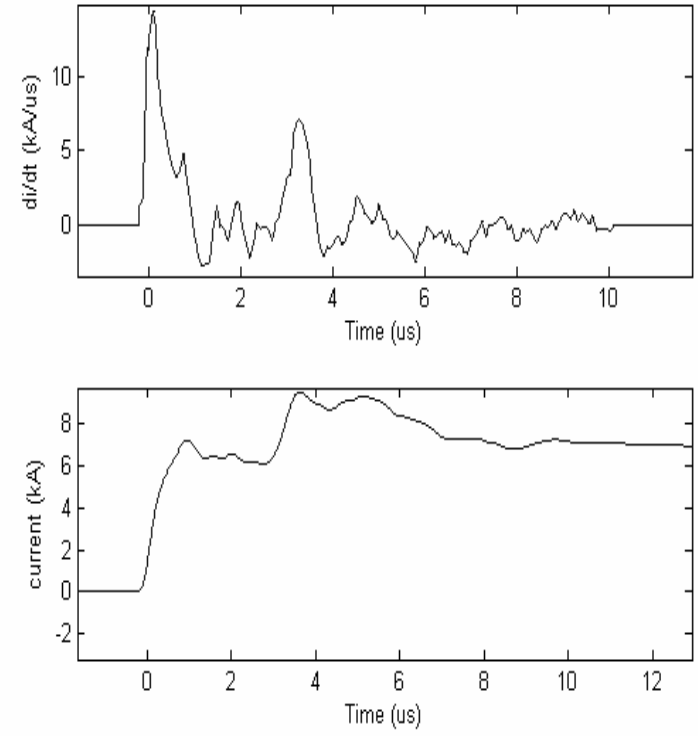

Figure 4. . Preliminary noise removal
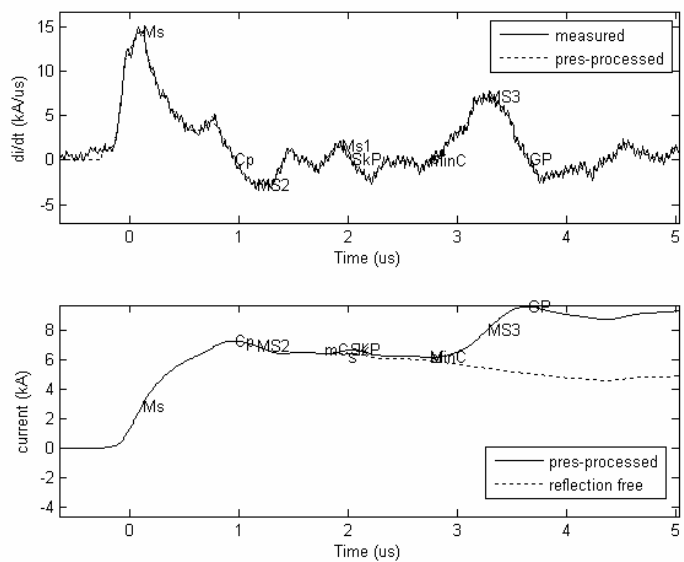

Figure 5. Peaks localisation and removal of the reflection signals

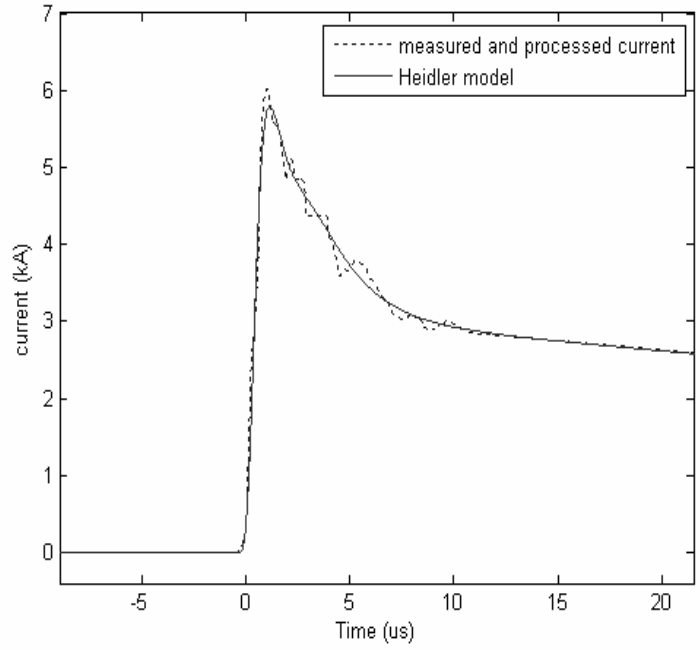

Figure 6. Heidler model of the lightning current

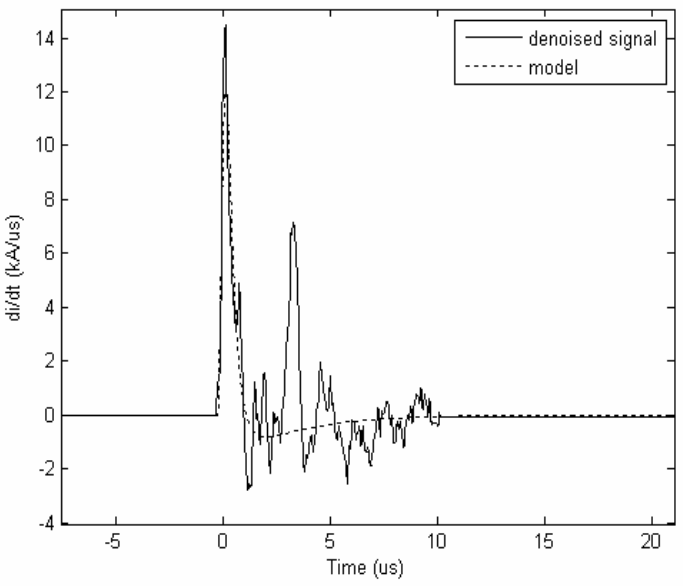

Figure 7. Modeled current derivative waveform 


\section{CONCLUSION}

After investigating all the ways used to overcome the problem of dependence of the denoising process on the signals on hand we have chosen the most adequate way that best tackles our problem.

The newly developed Divide and Conquer denoising method was inspired by two investigated signal optimization and denoising techniques. In the first place, we used the marching on the graph procedure in the Newton-Raphson optimization technique to find the optimum points of a signal, and to find the different variation points of the lightning derivative signal and its integral that allowed the elimination of most of the noise. Secondly, we imitated the basis pursuit method that chooses the best basis that represent a signal and removes the noise from it by optimizing its decomposition coefficients; by finding an appropriate model to the measured signal using the optimization tools and an approximated version (denoised) signal to the measured one. This new strategy divides the lightning current derivative according to its contents. It conquers its active part to remove the noise from the lightning current due to reflections and then calculates automatically the parameters of the two waveforms, and finally finds an optimized model with an infinite SNR for the current waveform, based on Heidler function.

The Divide-and-Conquer denoising technique have been evaluated by using Heidler models for different ranges of magnitude of the measured lightning current derivatives to which noise only signals, measured at the $\mathrm{CN}$ Tower in the absence of lightning, were added. For this purpose, more than 10 Heidler models corresponding to different ranges of current derivative waveforms, measured at the tower, have been generated. These current derivative models have been artificially noised by sequentially adding 25 different noise only signals collected at the tower. The noised models have been de-noised by the proposed algorithm. The results showed substantial improvement in the SNR of an average of $51 \mathrm{~dB}$ for the low signals range (2 to $10 \mathrm{kA} / \mu \mathrm{s})$, an average amelioration of $44 \mathrm{~dB}$ for the range of signals $(10 \mathrm{kA} / \mu \mathrm{s}$ to $25 \mathrm{kA} / \mu \mathrm{s})$ and an enhancement of $26 \mathrm{~dB}$ for the high level current derivatives above $25 \mathrm{kA} / \mu \mathrm{s}$. It also showed improvements of the Norm of an average of $73 \%$ for the low signals range ( 2 to 10 $\mathrm{kA} / \mu \mathrm{s})$, an average of $81 \%$ for the range of signals extending from $10 \mathrm{kA} / \mu \mathrm{s}$ to $25 \mathrm{kA} / \mu \mathrm{s}$ and an average of $51 \%$ for the high level current derivative above $25 \mathrm{kA} / \mu \mathrm{s}$. The average improvement for the correlation between the models and their de-noised signals was $76 \%$ for the low range signals, $93 \%$ for the medium range signals and $67 \%$ for the high level signals.

\section{REFERENCES}

[1] Martin A. Uman, "The Lightning Discharge," DoverPublication, Inc., Mineola, New York, 2001.

[2] A.M. Hussein, W. Janischewskyj, J.-S. Chang, V. Shostack, W.A. Chisholm, P. Dzurevych, and Z.-I. Kawasaki, "Simultaneous measurement of lightning parameters for strokes to the Toronto Canadian National Tower," Journal of Geophysical Research-Atmosphere, vol.100, no. 5, pp. 88538861, May 1995.

[3] W. Janischewskyj, A.M. Hussein, V. Shostak, I. Rusan, J.X. Li and J.S. Chang, "Statistics of Lightning Strikes to the
Toronto CN Tower (1978-1995)," IEEE Trans. Power Delivery, vol. 12, no. 3, pp. 1210-1221, July 1997.

[4] A.M. Hussein, W. Janischewskyj, M. Milewski, V. Shostak, J.S. Chang and W. Chisholm, "Current waveform parameters of CN Tower lightning return strokes," Journal of Electrostatics, Vol. 60, Nos. 2-4, pp. 149-162, March 2004.

[5] Ying Chen, "Wavelet Analysis And Statistics Of CN Tower Lightning Current Waveforms," M.E.Sc. Thesis, University of Weston Ontario, London, Ontario, 1997.

[6] P. Liatos and A.M. Hussein, "Characterization of Noise in the Lightning Current Derivative Signals Measured at the CN Tower," IEEE Transaction on Electromagnetic Compatibility (EMC), vol. 47, No. 4, pp. 986-997, November, 2005.

[7] Ouarda Nedjah, A.M. Hussein, R. Sotudeh and W. Janischewskyj, "Wavelet noise removal from CN Tower lightning current waveforms," International Signal Processing Conference, Paper 505 (pp. 1-6), Dallas, Texas, Mar. 31-Apr. 3, 2003.

[8] Ouarda Nedjah, A.M. Hussein, S. Krishnan and R. Sotudeh, "CN Tower Lightning Current Derivative Heidler Model for" the Validation of Wavelet De-Noising Algorithm," Proceedings, $18^{\text {th }}$ International Wroclaw Symposium and Exhibition on Electromagnetic Compatibility, pp. 282 - 287, Wroclaw, Poland, June 28 - 30, 2006.

[9] M.J. Islam and A.M. Hussein, "De-noising the $\mathrm{CN}$ Tower lightning current derivative signal using short term Fourier transform-based spectral subtraction," Proceedings of the 17th International Symposium and Exhibition on Electromagnetic Compatibility, pp. 400-406, Wroclaw, Poland, June 29-July 1, 2004.

[10] K. Bitner and A.M. Hussein, "Modelling of the CN Tower Lightning Return-Stroke Current Derivative," Proceedings of the $28^{\text {th }}$ International Conference on Lightning Protection (ICLP), pp. 261-266, Kanazawa, Japan, September 18-22, 2006.

[11] F. Heidler, J.M. Cvetic, B.V. Stanic, "Calculation of lightning Current Parameters", IEEE transaction on Power delivery, Vol. 14, No. 2, pp. 399-404, April 1999.

[12] R. R. Coifman and M. V. Wickerhauser, "Entropy-based algorithms for best-basis selection," IEEE Trans. Inform. Theory, Vol. 38, pp. 713-718, 1992

[13] S. Mallat and Z. Zhang, "Matching pursuit in a timefrequency dictionary," IEEE Trans. Signal Proc., 41 (1993), pp. 3397-3415.

[14] Jorma Rissanen, "MDL Denoising," IEEE Transactions on Information Theory, vol, 46, No.7, pp.2537-2543 November 2000.

[15] S. Chen, D. Donoho, and M. Saunders, "Atomic Decomposition by Basis Pursuit," SIAM Review, 43, pp. 129159, 2001

[16] Philip Egill, Walter Murray, Dulce B. Ponceleon, M. Saunders, "Solving Reduced KKT Systems in Barrier methods for linear and Quadratic programming," Technical report, SOL 91-7, July 1991.

[17] Greg Astfalk, Irvin Lustig, Roy Marsten, David Shanno, "The interior-point method for Linear Programming," IEEE software, pp. 61-68, July 1992.

[18] J.L Bermudez, C.A. Pena-Reyes, "Use of Genetic Algorithms to Extract Primary Lightning Current Parameters," $5^{\text {th }}$ International Symposium on EMC, Sorrento, Italy, pp.241246, September, 9-13, 2002 